\title{
Importance of self-interaction-error removal in density functional calculations on water cluster anions
}

\author{
Jorge Vargas, ${ }^{a \dagger}$ Peter Ufondu, ${ }^{\dagger}$ Tunna Baruah, ${ }^{\dagger}, \Phi^{\top}$ Yoh Yamamoto, ${ }^{\dagger}$ Koblar A. \\ Jackson, ${ }^{\ddagger}$ and Rajendra R. Zope ${ }^{*, \dagger, \uparrow}$ \\ $\dagger$ Department of Physics, The University of Texas at El Paso, El Paso, Texas, 79968 \\ $\ddagger$ Physics Department and Science of Advanced Materials Program, Central Michigan \\ University, Mt. Pleasant Michigan, 48859 \\ I Computational Science Program, The University of Texas at El Paso, El Paso, Texas, \\ 79968 \\ E-mail: rzope@utep.edu
}

\footnotetext{
${ }^{a}$ Current address: Unidad Academica de Fisica, Universidad Autonoma de Zacatecas, Calz. Solidaridad Esq. Paseo de la Bufa S N, Zacatecas, Mexico. C.P. 98060
} 


\begin{abstract}
Accurate description of the excess charge in water cluster anions is challenging for standard semi-local and (global) hybrid density functional approximations (DFAs). Using the recent unitary invariant implementation of the Perdew-Zunger self-interaction correction (SIC) method using Fermi-Löwdin orbitals, we assess the effect of selfinteraction error on the vertical detachment energies of water clusters anions with the local spin density approximation (LSDA), Perdew-Burke-Ernzerhof (PBE) generalized gradient approximation, and the strongly constrained and appropriately normed (SCAN) meta-GGA functionals. Our results show that for the relative energies of isomers with respect to reference $\operatorname{CCSD}(\mathrm{T})$ values, the uncorrected SCAN functional has the smallest deviation of $21 \mathrm{meV}$, better than that for the MP2 method. The performance of SIC-SCAN is comparable to that of MP2 and is better than SIC-LSDA and SIC-PBE, but it reverses the ordering of the two lowest isomers for water hexamer anions. Removing self interaction error (SIE) corrects the tendency of LSDA, PBE, and SCAN to over-bind the extra electron. The vertical detachment energies (VDEs) of water cluster anions, obtained from the total energy differences of corresponding anion and neutral clusters, are significantly improved by removing self-interaction and are better than the hybrid B3LYP functional, but fall short of MP2 accuracy. Removing SIE results in substantial improvement in the position of the eigenvalue of the extra electron. The negative of the highest occupied eigenvalue after SIC provides an excellent approximation to the VDE, especially for SIC-PBE where the mean absolute error with respect to $\operatorname{CCSD}(\mathrm{T})$ is only $17 \mathrm{meV}$, the best among all approximations compared in this work.
\end{abstract}

\title{
Introduction
}

The hydrated electron is a system that has long attracted the attention of the scientific community ${ }^{116}$ due to its importance in chemical and biological processes, such as atmospheric chemistry $^{7}$ or radiation damage in $\mathrm{DNA}, \frac{8}{8}$ to name just two. In this context, systematic 
study of water cluster anions can be useful for obtaining insights into the behavior and evolution of electron hydration. Indeed, since the first observation of a free hydrated electron in the gas phase ${ }^{9}$, a large number of studies have been performed on water cluster anions resulting in debate about whether the extra electron is bound in a delocalized surface state or bound internally in a cavity. $\frac{10}{15}$ Even before its first observation, theoretical models for the hydrated electron were proposed $16 \mid 17$. Later, improvements in experimental techniques allowed direct comparison with theoretical results. Photoelectron spectroscopy 18 and vibrational spectroscopy $25+29$ the structural and electronic properties of water cluster anions. These experiments have provided data for the vertical detachment energy (VDE) of small water clusters. The VDE is the energy required to remove an excess electron from an anion. Moreover, a combination of simulations and experimental vibrational spectra has been used to identify the structure of stable isomers ${ }^{26|30| 31}$. It is, however, in general difficult to assign a particular isomer to the observed experimental spectra as the experiments usually sample non-equilibrium ensembles of clusters. A detailed description of the challenges involved in ascribing specific spectroscopic features to an individual isomer of $\left(\mathrm{H}_{2} \mathrm{O}\right)_{6}^{-}$is provided by Choi and Jordan in Ref. 32,

Accurate description of a hydrated electron poses a significant challenge to density functional approximations (DFAs). In general, DFAs are inadequate for describing the binding of the excess electron. As a result, most computational studies on small anionic water clusters $\frac{10|19| 30 \mid 33}{43}$ have used a post Hartree-Fock (HF) method like the Møller-Plesset perturbation method (MP2) or the coupled cluster method with single, double and perturbative triple excitations $\operatorname{CCSD}(\mathrm{T})$. CCSD $(\mathrm{T})$ is computationally very demanding and has only been used to study small clusters and often in these studies, structural relaxation is carried out by a faster method like MP2.

Many failures of the DFAs have been ascribed to the self-interaction error (SIE) present in the approximate exchange-correlation functionals. The SIE occurs due to the incomplete 
cancellation of the self-Coulomb by the approximate self-exchange energy. This error is particularly dominant in the local and semi-local approximations and is mitigated to some extent in the hybrid DFAs due to the addition of HF exchange. It has, however, been found that the most popular global hybrid functional B3LYP ${ }^{44}$ significantly overestimates the VDEs of anionic water clusters. On the other hand, a recently proposed non-empirical meta-GGA functional (SCAN) ${ }^{45}$ has been found to provide an excellent description of the structural, electronic, and dynamic properties of liquid water ${ }^{46}$. The SCAN meta-GGA functional, unlike most other DFAs, also predicts the energetics of gas-phase water hexamers and ice phases with quantitative accuracy ${ }^{45}$.

In this work we examine the role of SIE in three non-empirical functionals that belong to the lowest three rungs of Jacob's ladder on the VDEs of water. The functionals used here are the local density approximation (LDA), generalized gradient approximation (GGA) given by Perdew, Burke, Ernzerhof (PBE) ${ }^{47}$, and the SCAN meta-GGA functional ${ }^{455}$. The incorrect asymptotic form of the DFA potential caused by SIE is expected to have a particularly strong impact on the description of anionic systems which typically have a weakly bound extra electron in a diffuse orbital. We explictly remove SIE using the self-interaction correction (SIC) applied to the DFA's. We study both the effect of SIC on the orbital energy of the highest occupied electron orbital and on the total energy difference between corresponding anion and neutral systems. We also check the error made by using the SCAN functional for calculating the VDE. The details of the self-interaction correction method and the computation scheme is presented in the next section followed by the results and discussion.

\section{Methodology}

In 1981, Perdew and Zunger proposed an orbital-wise correction to remove the self-interaction error ${ }^{48}$ in density functional approximations. In the Perdew-Zunger self-interaction correc- 
tion (PZSIC) method the exchange-correlation energy is corrected as

$$
E_{\mathrm{XC}}^{\mathrm{SIC}}\left[\rho_{\uparrow}, \rho_{\downarrow}\right]=-\sum_{i, \sigma}^{N_{\sigma}}\left\{U\left[\rho_{i \sigma}\right]+E_{\mathrm{XC}}\left[\rho_{i \sigma}, 0\right]\right\},
$$

where $i$ runs over the $N_{\sigma}$ occupied orbitals of spin $\sigma$, and $\rho_{i \sigma}$ is the $i^{\text {th }}$ orbital density. The terms $U\left[\rho_{i \sigma}\right]$ and $E_{\mathrm{XC}}\left[\rho_{i \sigma}, 0\right]$ are the exact self-Coulomb and approximate self exchange correlation $(\mathrm{XC})$ energies, respectively. The correction vanishes when $E_{\mathrm{XC}}$ is the exact $\mathrm{XC}$ functional. Pederson et al. have shown that the orbitals minimizing the PZ-SIC total energy must satisfy the conditions known as the localization equations: $\frac{49 \mid 50}{4}$

$$
\left\langle\phi_{j \sigma}\left|V_{j \sigma}^{\mathrm{SIC}}-V_{i \sigma}^{\mathrm{SIC}}\right| \phi_{i \sigma}\right\rangle
$$

where $V_{i \sigma}^{\text {SIC }}$ is the SIC potential for the $\mathrm{i}^{\text {th }}$ orbital. Satisfying the localization equations is a computationally slow process and the self-interaction corrected energy obtained as a result is not guaranteed to be size-consistent.

A recent scheme for SIC proposed by Pederson, Ruszinzsky and Perdew ${ }^{51}$ circumvents the need for satisfying Eq. 22. The localized orthonormal set of orbitals is derived from Fermi orbitals which depend on the density matrix and the spin density as:

$$
\phi_{i \sigma}^{\mathrm{FO}}(\mathbf{r})=\frac{\sum_{j} \psi_{j \sigma}^{*}\left(\mathbf{a}_{i \sigma}\right) \psi_{j \sigma}(\mathbf{r})}{\sqrt{\sum_{j}\left|\psi_{j \sigma}\left(\mathbf{a}_{i \sigma}\right)\right|^{2}}}=\frac{\gamma_{\sigma}\left(\mathbf{a}_{i \sigma}, \mathbf{r}\right)}{\sqrt{\rho_{\sigma}\left(\mathbf{a}_{i \sigma}\right)}},
$$

where $\gamma_{\sigma}\left(\mathbf{a}_{i \sigma}, \mathbf{r}\right)$ is the single-particle density matrix of the KS system, and $\mathbf{a}_{i \sigma}$ are a set of points in real space called the Fermi orbital descriptors (FODs). The Fermi orbitals are normalized, but are not othogonal. They are orthogonalized using Löwdin's method of symmetric orthonormalization ${ }^{\frac{52}{2}}$ resulting in an orthonormal set of local orbitals called Fermi-Löwdin orbitals (FLOs). The positions of the FODs determine the Fermi orbitals and different choices lead to different total energies. The optimal positions of the FODs are obtained in a procedure that is analogous to a molecular geometry optimization. The 
gradients of the energy with respect to the FODs can be calculated $\underline{53154}$ and used in a preconditioned limited-memory Broyden, Goldfarb, Shanno (LBFGS) algorithm 5 . The FODs are updated after each self-consistent FLOSIC calculation. The optimization is carried out until the forces on all FODs drop below 0.0001 Ha/Bohr.

The Fermi-Löwdin orbital based self-interaction correction (FLO-SIC) method is implemented in the FLOSIC code $\mathrm{e}^{56157}$ that is based on the UTEP-NRLMOL code ${ }^{58159}$. This code uses a Gaussian basis set $\underline{60}$, and a variational integration mesh $\underline{61}$ to perform numerically precise calculations on molecules composed of non-relativistic atoms. The FLOSIC implementation has been used to study a number of different properties for systems ranging in size from atoms $\underline{62[63}$ and small molecules to larger molecules such as $\mathrm{Mg}$-porphyrin and $\mathrm{C}_{60} \underline{64}$. FLOSIC has been used to study various properties ranging from energetic properties such as

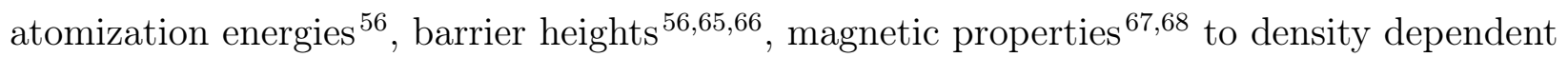
properties such as dipole moment $\frac{69}{6}$ and polarizability ${ }^{70}$.

We use the default Pederson-Porezag NRLMOL basis ${ }^{60}$ that is specially optimized for the Perdew-Burke-Ernzerhof (PBE) ${ }^{47}$ generalized gradient approximation (GGA). The calculations are spin-polarized for systems with net spin. To obtain an accurate description of water anions, extra diffuse functions are added to account for the more diffuse charge distribution

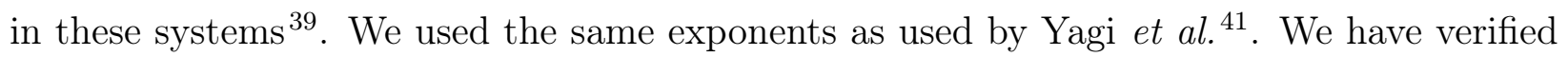
that these exponents give converged results for the VDEs. The exponents are $9.87 \times 10^{-3}$ au, $8.57 \times 10^{-3} \mathrm{au}$, and $3.72 \times 10^{-3}$ au for oxygen $s, p$, and hydrogen $s$ respectively.

Yagi and coworkers reported the anionic water cluster geometries optimized at the MP2 level which they used subsequently to perform the $\operatorname{CCSD}(\mathrm{T})$ calculations 1 . To facilitate a direct comparison with the earlier MP2 and $\mathrm{CCSD}(\mathrm{T})$ results, we used the same set of MP2 optimized geometries from Ref. 41 in our calculations.

Each water molecule has five electrons of each spin. The FODs representing the valence electrons of each molecule form a tetrahedral structure with the center at the oxygen atom and two of the vertices along the two O-H bonds. The tetrahedral structure can be seen 
in Fig. 1. The FOD representing the oxygen core orbital is found to be at the oxygen nuclear position. The extra FOD for the anions is initially placed at the central region of the singly occupied molecular orbital obtained at the PBE level. The position of this FOD is then optimized along with all the others using the pre-conditioned LBFGS routine outlined above.

The VDE can be calculated as the total energy difference between the energy of the anion and neutral cluster at the geometry of the anion. The negative of the energy of the highest occupied molecular orbital (HOMO) also mimics the negative of the electron removal or detachment energy ${ }^{71-74}$. The HOMO eigenvalue for an anion in a DFA calculation is generally found to be positive, corresponding to an unbound outer electron. Therefore the detachment energies can only be calculated from total energy differences when using DFAs. As shown below, the anion HOMO in FLOSIC-DFA calculations is negative and a good approximation to the removal energy. We examine the VDEs calculated from total energies as well as from the HOMO eigenvalues for the FLOSIC calculations.

\section{Results and discussion}

The anionic water clusters contain a weakly bound extra electron. The 20 water isomers $\left(\mathrm{H}_{2} \mathrm{O}\right)_{n}^{-}$in the range $n=2-6$ studied here can be grouped by various types of extra electron binding motifs. The clusters studied here and other slightly different clusters have been reported in several studies with different names ${ }^{10119134137422}$. For direct comparison with

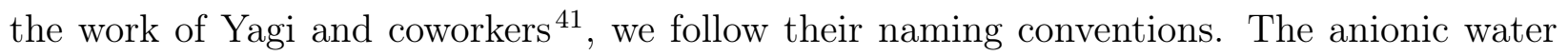
clusters are classified as linear(L), double acceptor (AA), donor(D), and internal (I). In linear (L) clusters the water molecules are bound by successive hydrogen bonds (HB). These structures tend have the smallest number of HBs among all the isomers. The AA type clusters have one double-acceptor water molecule such that its hydrogen atoms not involved in any HB. In the D type clusters the extra electron is bound collectively by dangling O-H 
bonds. In I type anions the extra electron is trapped internally, as in a cavity.

The water clusters studied here are presented in figures 1-5, where the positive electron density difference (EDD) between the anionic and the neutral systems shows the charge density of the extra electron. The density difference also contains rearrangements of the neutral molecule density due to the presence of the extra electron, but such differences are insignificant compared to density of the extra electron itself. The FLOSIC calculations of the water anions were started with the same FOD positions as for the neutral water cluster but with one extra FOD. All the FODs are fully re-optimized for the anionic clusters. For simplicity, only the optimized extra FOD position is shown in Figs 2-5 and an image including all the FODs is shown for the anionic water dimer in Fig. 1.

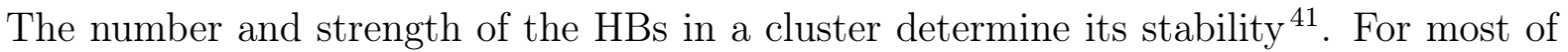
the water cluster sizes studied here, the isomers with dangling $\mathrm{O}-\mathrm{H}$ bonds (D) are the lowest energy structures. The D structures are cyclic, with every water molecule being both donor and acceptor of a hydrogen bond and the dangling $\mathrm{O}-\mathrm{H}$ bonds oriented toward the same direction where most of the extra charge is accommodated 41 . Moreover, it was shown that the orientations of the dangling $\mathrm{O}-\mathrm{H}$ bonds in most stable anionic clusters are different from

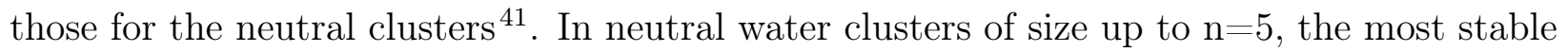
isomers have the dangling $\mathrm{OH}$ bonds on alternating sides of a quasi-planar polygon 35137 .

In the neutral water dimer, the torsional angle between the bisecting axis of the proton acceptor and the line segment connecting the oxygens is experimentally found to be $57^{\circ} \mathbf{7 5}$. Kim et al. studied several configurations of the water dimer $\stackrel{35}{35}$ at the CCSD(T) level. They concluded that the presence of the extra electron in the dimer introduces a large change in the torsional angle. They also concluded that the anionic water dimer is a very floppy structure with large vibrational zero-point energy effects. Among the reported structures of the neutral water dimers, the trans isomer has a dipole moment of $2.7 \mathrm{D}$ whereas nearly isoenergetic cis isomer has a dipole moment of 4.3D. The individual dipoles of the water molecules are almost parallel in the cis configuration. Thus, although the total energies of 
Table 1: Relative energies in meV of the different water cluster anions with respect to the $\mathrm{D}$ isomers. The dipole moment in Debye, $\mu$, is from the SICSCAN calculations of the neutral clusters. The mean absolute deviation (MAD) is with respect to the $\operatorname{CCSD}(\mathrm{T})$ values.

\begin{tabular}{lcccccccccc}
\hline \hline \multirow{2}{*}{ Cluster } & \multirow{2}{*}{$\mathrm{CCSD}^{*}(\mathrm{~T})^{a}$} & \multirow{2}{*}{$\mathrm{MP}^{a}$} & \multirow{2}{*}{ B3LYP $^{a}$} & \multicolumn{3}{c}{ DFA } & \multicolumn{5}{c}{ FLOSIC } \\
\hline 3D & - & - & - & - & - & - & - & - & - & 4.03 \\
3L & 69 & 78 & 9 & 99 & 45 & 92 & 145 & 111 & 111 & 7.13 \\
3AA & 147 & 173 & 87 & 282 & 131 & 169 & 248 & 156 & 177 & 6.40 \\
3I-2 & 178 & 204 & 113 & 304 & 144 & 185 & 271 & 174 & 197 & 6.39 \\
3I-1 & 533 & 551 & 343 & 784 & 407 & 512 & 714 & 461 & 503 & 1.75 \\
4D & - & - & - & - & - & - & - & - & - & 5.17 \\
4AA & 134 & 178 & 121 & 329 & 185 & 180 & 282 & 208 & 180 & 8.92 \\
4L & 191 & 208 & 134 & 375 & 194 & 224 & 317 & 215 & 199 & 9.39 \\
4I & 425 & 451 & 308 & 706 & 375 & 429 & 656 & 469 & 471 & 0.00 \\
5D & - & - & - & - & - & - & - & - & - & 5.39 \\
5AA-2 & -30 & 30 & 43 & 67 & 84 & -23 & 104 & 149 & -7 & 9.03 \\
5AA-1 & 139 & 186 & 134 & 305 & 177 & 165 & 294 & 217 & 88 & 9.48 \\
5L & 204 & 225 & 147 & 422 & 228 & 242 & 357 & 241 & 225 & 10.77 \\
5I & 152 & 208 & 139 & 268 & 157 & 127 & 310 & 268 & 165 & 8.71 \\
6D & - & - & - & - & - & - & - & - & - & 5.51 \\
6AA-2 & -56 & -13 & -9 & 21 & 16 & -51 & 70 & 94 & 13 & 10.41 \\
6AA-1 & 117 & 182 & 126 & 248 & 151 & 106 & 317 & 291 & 210 & 10.24 \\
6L & 351 & 356 & 252 & 633 & 327 & 387 & 544 & 345 & 377 & 13.09 \\
6I & 516 & 533 & 382 & 906 & 446 & 524 & 864 & 596 & 619 & 0.00 \\
\hline MAD & - & 32 & 66 & 383 & 204 & 21 & 168 & 73 & 39 & 0.30 \\
\hline \hline
\end{tabular}

${ }^{a}$ Ref. 41

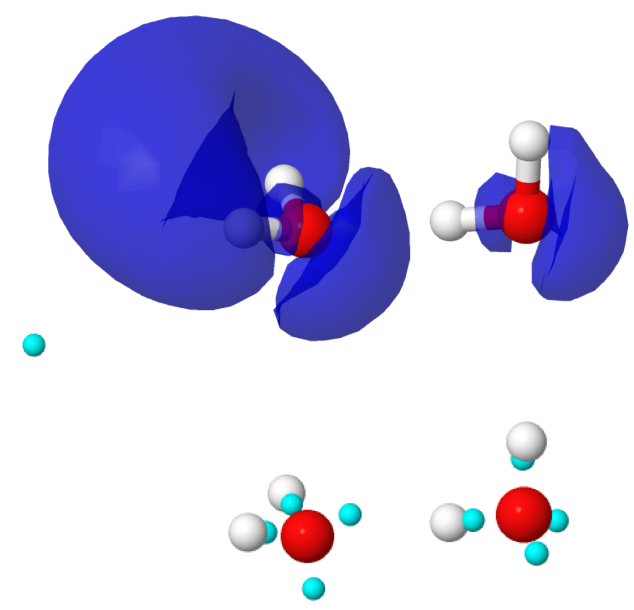

Figure 1: (Top)Electron density difference (blue) of anionic water dimer anion and (bottom) the optimized FODs shown as cyan spheres. 
the two anions are very close, it was shown ${ }^{35}$ that the trans isomer barely binds an extra electron, while the cis isomer has significantly more binding due to its larger dipole. Based on these earlier reports, FLOSIC calculations were performed on the cis isomer. In Fig. 1 the EDD of the cis isomer (2L) and the positions of the FODs are shown. As mentioned above, the FODs corresponding to the valence electrons form a tetrahedron around each oxygen atom and the extra FOD for the anion finds its optimal place at the $\angle \mathrm{H}-\mathrm{O}-\mathrm{H}$ bisector, but far away $(4.4 \AA)$ from the acceptor molecule. The figure shows the location of the extra electron density in the anion which is in the same direction as the extra FOD. A similar pattern is observed in all the other clusters. For that reason, only the extra FOD is shown in figures $2-5$, together with the EDD.

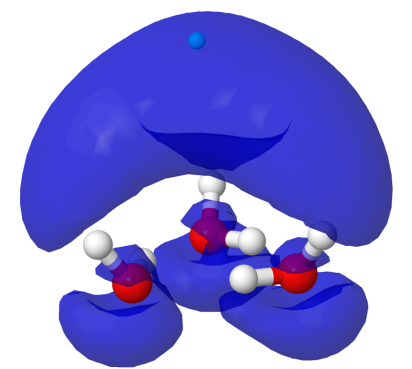

3D
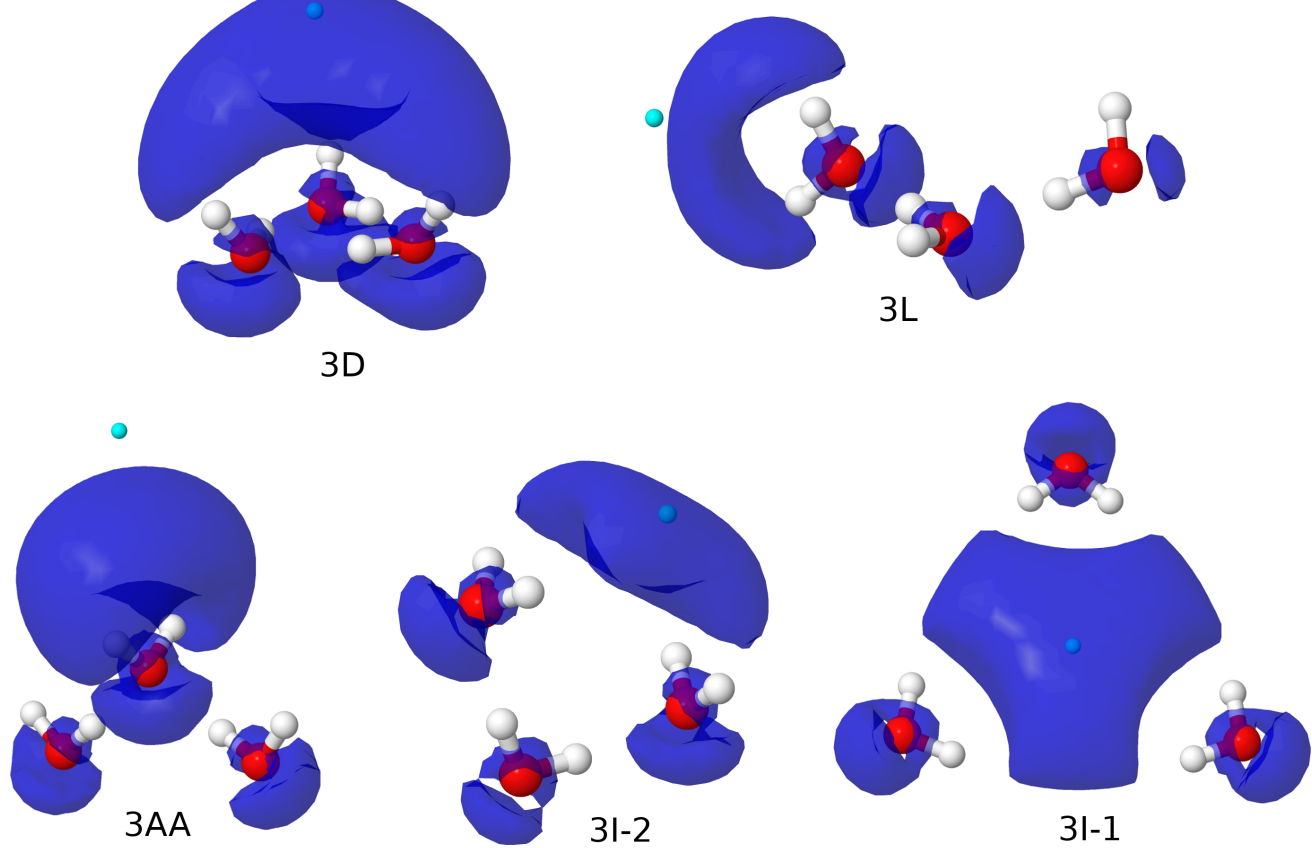

$31-1$

Figure 2: Water trimer anions with the EDD in blue and the optimized extra FOD of each isomer.

The EDD plots of the various trimer, tetramer, pentamer and hexamer isomers are shown in Fig. 2 - 5. The plots corresponding to various functionals are similar and therefore we show only the FLOSIC-LDA version. The FLOSIC EDD plots are in close agreement with the plots shown in Ref.41. The D structures show a collective cloud of the extra electron 
spread over multiple water molecules. From the trimer to the pentamer, the D structures have the water monomers forming a quasi-planar structure, but in case of the hexamers, the monomers in the D structure form a 3-dimensional book structure. However, for all sizes, the excess electron cloud is spread over the whole structure. In all the linear isomers, there is one only-donor and one only-acceptor water molecule with the intermediate molecules being both a donor and an acceptor of a HB. The excess charge of the anion is mainly accommodated in front of the dangling hydrogen atoms of the only-acceptor molecule. On the other hand, the double acceptor isomers have one molecule which attracts the excess electron cloud. In the case of the hexamers, two such double acceptor structures were examined. In the 6AA-1 isomer, there are two water molecules that are both double-acceptors. These two molecules contribute equally to accommodate the extra charge in 6AA-1, whereas in the 6AA-2 isomer the extra electron is mainly located around the only double acceptor water molecule. The I structures with internally trapped electrons are found to be higher in energy for the sizes under study here. The 3I-1 has D3h symmetry and the 4I structure has a center of inversion and, therefore, both have zero net dipole. Both 3I-2 and 5I structures have two water molecules that form a "bridge" for the extra charge.

The EDD plots mainly show the extra electron density as a diffuse cloud. We find that the extra FOD in the anionic cluster generally follows the cloud and is located away from the molecular framework. Our calculations also show that the dipole of the neutral cluster plays a minor role in the binding of the extra charge. As seen from Table 1, the dipole moment of the lower-energy clusters are in fact smaller than those of many high-lying isomers. In general, the linear (L) structure, which has a chain of HBs, has a larger dipole moment compared to the ring-like D structure.

The relative energies of the anionic water clusters with $n=3-6$ are presented in Table 1 . For the sizes $\mathrm{n}=3$ and 4 , the relative energies follow similar trends across all methods used here, including the FLOSIC-DFAs. The D structures of the trimer and tetramer are found to be the lowest energy isomer by all the methods (Cf. Table 1). The D structures are ring-like 
for both sizes. For the trimers, all the computational methods also put the linear isomer $(3 \mathrm{~L})$ as the second lowest isomer. However, Hammer et al. ${ }^{30}$ identified the linear structure for the trimer anion from infrared spectrum and theoretical calculations on the vibrational spectra of the trimer anion. Similarly, the 4AA isomer has been experimentally identified for the tetramer. ${ }^{21}$. Shin et al. also found weaker signals in their photo-electron spectra that can be ascribed to $4 \mathrm{~L}$ and $4 \mathrm{D}$, but the major presence of the $4 \mathrm{AA}$ isomer was unequivocally confirmed through IR experiments and B3LYP vibrational spectra comparisons ${ }^{10}$. The 5D isomer, which is presumably optimized from the $C_{5}$ ring with the five oxygen atoms forming a planar pentagon, does not conform to a planar structure upon optimization. One water molecule moves out-of-plane, while the two adjacent molecules reorient their dangling hydrogen atoms radially outward thus diminishing the characteristic collective cloud of the D isomers. From the pentamer onward, the D structure is not the lowest energy isomer at the $\operatorname{CCSD}(\mathrm{T})$ level. The same ordering is also seen with the SCAN functional.
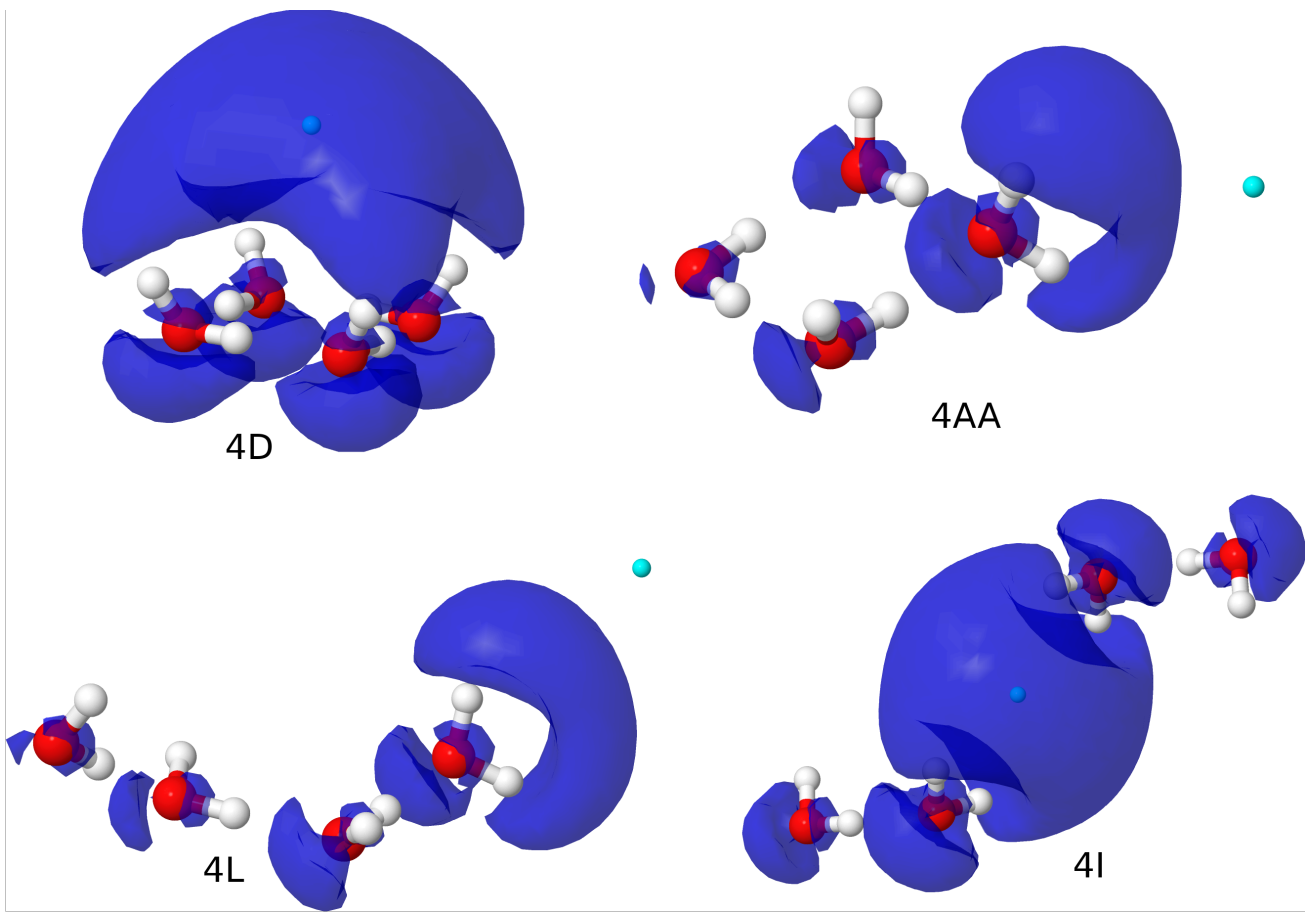

Figure 3: Water tetramer anions with the EDD in blue and the optimized extra FOD of each isomer.

Since the first experiments on the water clusters, the hexamer was identified as a magic 

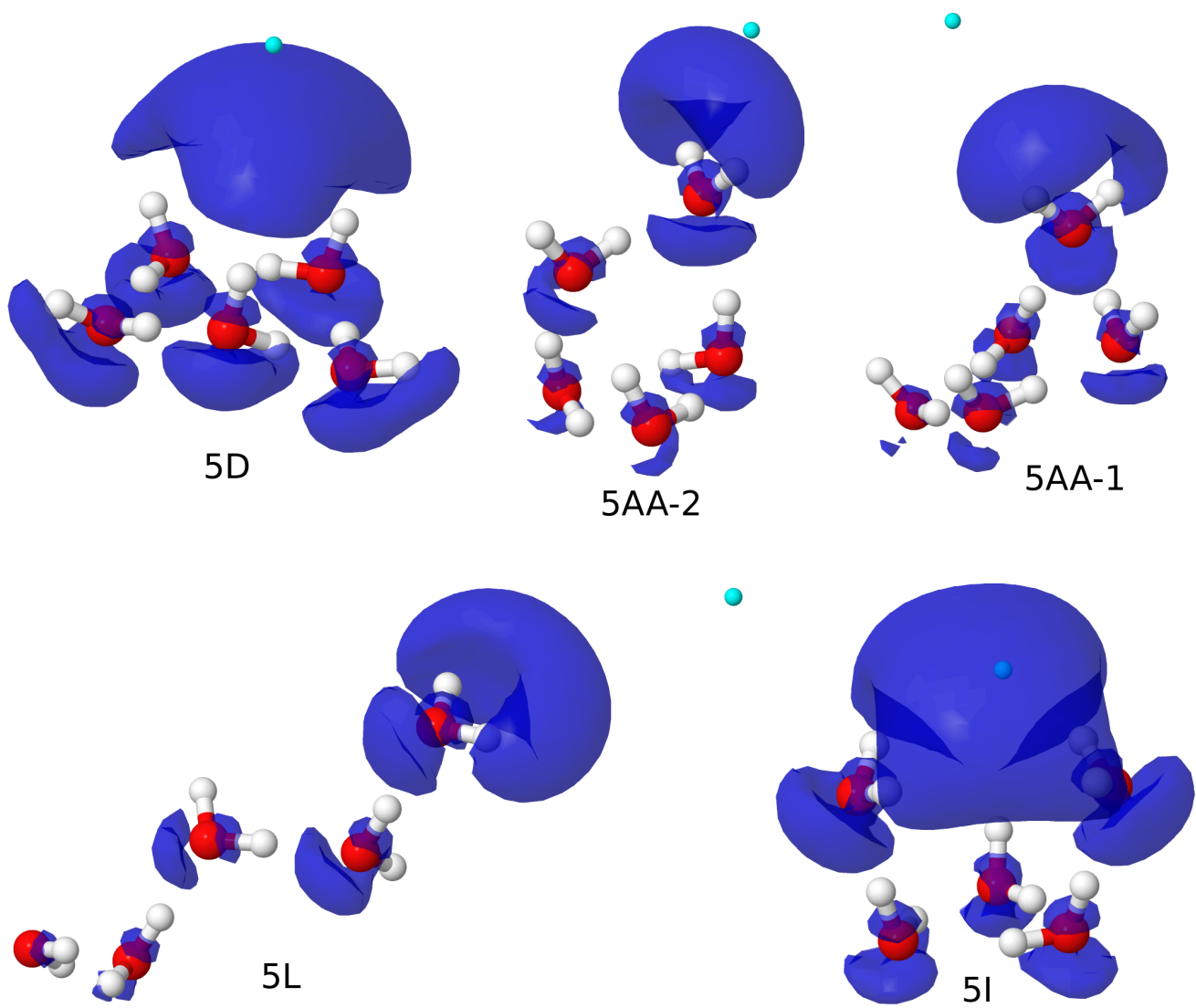

Figure 4: Water pentamer anions with the EDD in blue and the optimized extra FOD of each isomer. 

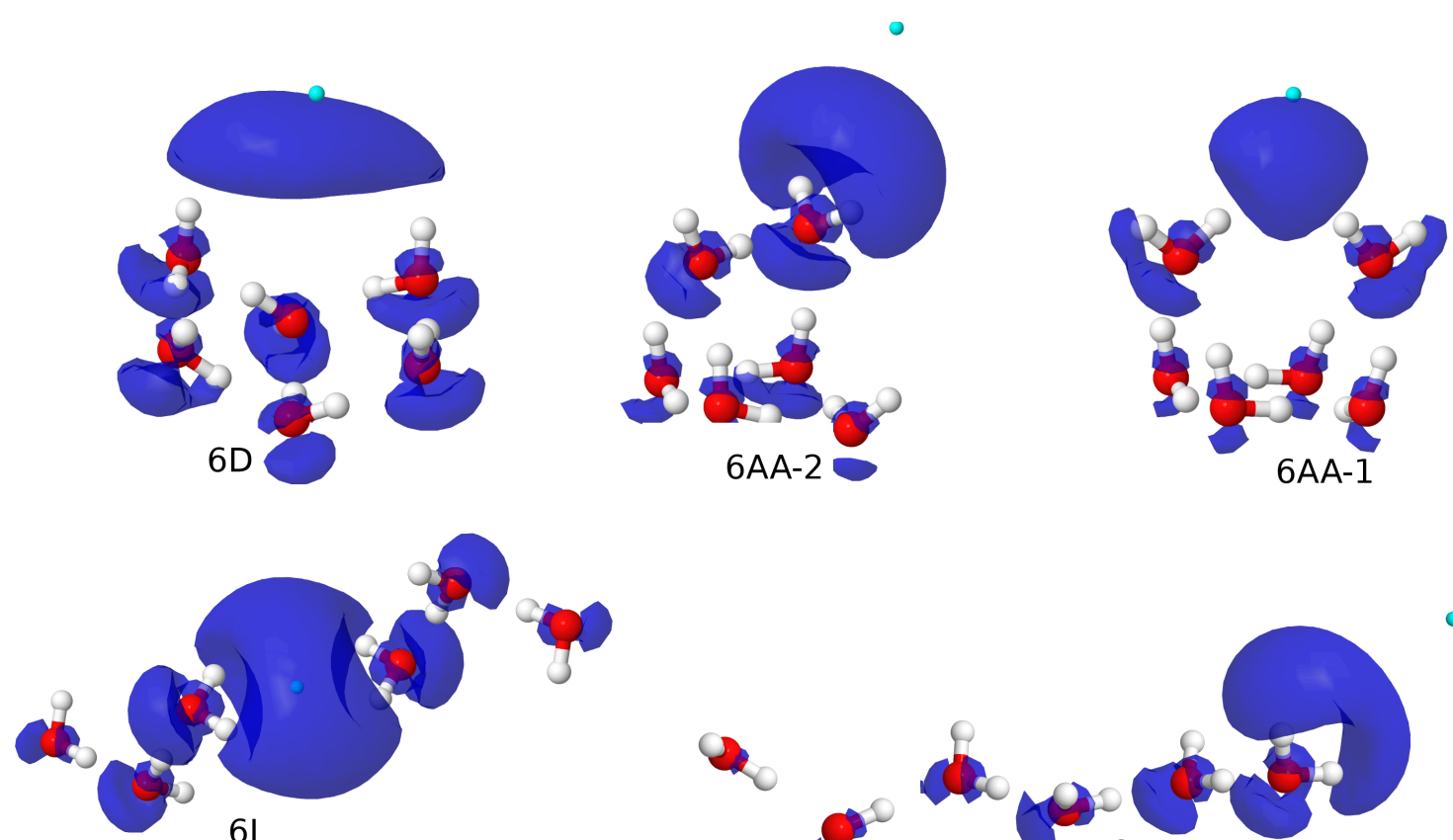

61

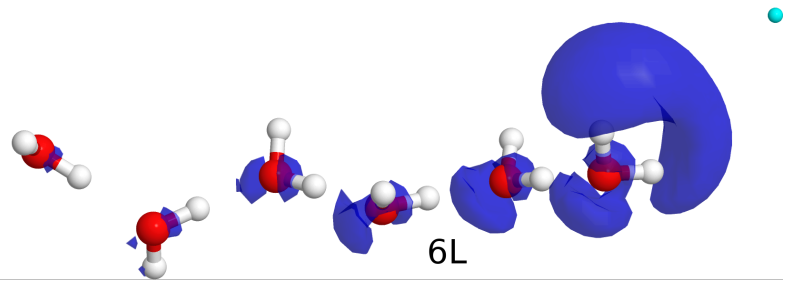

Figure 5: Water hexamer anions with the EDD in blue and the optimized extra FOD of each isomer.

number for the water cluster anions ${ }^{9 \mid 18}$ and has been extensively studied. It is broadly accepted that the experimental signal comes mainly from the 6AA-2 isomer with some contribution of the $6 \mathrm{D}^{25131 / 37}$. The $\operatorname{CCSD}(\mathrm{T})$ as well as MP2 and B3LYP calculations from Ref. 41 predict the 6AA-2 structure as the lowest energy isomer. The SCAN meta-GGA functional makes the same prediction. On the other hand, the FLOSIC method with all three different functionals predicts the $6 \mathrm{D}$ isomer to be the lowest energy structure. The $6 \mathrm{D}$ is the so-called book structure and the collective cloud is more concentrated on the two upper molecules as depicted in Fig. 5. The 6AA-2 can be thought of as a combination of the 4D isomer and the water dimer. The 6AA-1 and 6AA-2 structures are related to the so-called prism structure, which is one of the most stable neutral hexamers. The difference is that in the structure of the anions, the prism has one broken edge. In the 6AA-1 isomer there are two double acceptor molecules without any HB between them. These two molecules contribute equally to accommodate the extra charge in 6AA-1, whereas in the 6AA-2 isomer the extra electron is mainly located around the single double acceptor water molecule. 6AA-2 is favored over 
6AA-1 by CCSD(T), MP2, B3LYP, and the SCAN functional. The relative energies of these double acceptor clusters are comparable in $\operatorname{SCAN}$ and $\operatorname{CCSD}(\mathrm{T})$. The $6 \mathrm{~L}$ and $6 \mathrm{I}$ isomers, with the largest and smallest dipole moments, respectively, have significantly higher energies in all methods and are not likely to be found experimentally. Although the isomer ordering of the hexamers using SCAN agrees with that of $\operatorname{CCSD}(\mathrm{T})$, the SIC-SCAN results show same ordering as for SIC-LSDA and SIC-PBE.

Overall, the SCAN meta-GGA functional results for the stability of the anionic clusters are in close agreement with the $\operatorname{CCSD}(\mathrm{T})$ predictions. The ordering of all the isomers is the same and the mean absolute deviation (MAD) of relative energies is only $21 \mathrm{meV}$. The energy ordering of isomers for the smaller anionic clusters containing 3 and 4 water molecules is the same for all three functionals and their FLOSIC counterparts. For $n=5$ and 6 , LDA and PBE and FLOSIC-LDA and FLOSIC-PBE all invert the ordering of the two lowest-energy isomers. Both SCAN and FLOSIC-SCAN give the correct order for these isomers for $\mathrm{n}=5$, but only SCAN does for $n=6$. We also find that the deviation of the relative energies from the $\operatorname{CCSD}(\mathrm{T})$ values is reduced significantly upon removal of self-interaction error from LDA and PBE. For LDA, the MAD decreases from $383 \mathrm{meV}$ to $168 \mathrm{meV}$, and for PBE, from 204 $\mathrm{meV}$ to $73 \mathrm{meV}$. On the other hand, correcting for self-interaction in SCAN increases the MAD from $21 \mathrm{meV}$ to $39 \mathrm{meV}$. The performance of the SCAN functional is the best among all approximations including MP2. This observation is consistent with earlier results where the SCAN functional was found to provide an excellent description of the ordering ${ }^{45576}$ of the neutral water hexamers, as well as the ordering of various phases of ice. It should be noted that SCAN is a meta-GGA functonal whose correlation component is already self-interaction free. Removal of SIE from SCAN results in over-correcting, resulting in a somewhat degraded performance. However, FLOSIC-SCAN results are still comparable to MP2. 


\section{Vertical detachment energies}

The VDEs calculated from total energy differences between neutral and anionic clusters with and without FLOSIC are presented in Table2. Accurate description of the VDE is a challenge for the density functional approximations due to the inherent self-interaction present in these DFAs. Presence of SIE, in general, results in excessive electron delocalization causing significant errors in the electron binding energies. Indeed, as can be seen from the Table 2. the errors made by the pure DFAs are substantial. Since the CCSD $(T)$ values are in excellent agreement with the available experimental values and also because the $\operatorname{CCSD}(\mathrm{T})$ values are available for all the clusters studied here, we calculate the errors in DFAs and SIC-DFAs with respect to the CCSD(T) results. The mean absolute error (MAE) in VDE with respect to $\operatorname{CCSD}(\mathrm{T})$ results are 339,247 , and $127 \mathrm{meV}$ for LSDA, PBE, and SCAN, respectively. These numbers highlight the tendency of these functionals to over-bind an extra electron to water clusters, but they also show that as one climbs the Perdew-Schmidt Jacob's ladder 77 of increasingly complex functionals, the tendency decreases. The fourth rung of functionals corresponds to the hyper-GGA functionals. The hybrid functionals that include a certain fraction of HF exchange belong to this rung. In many situations, HF calculations have errors that are opposite to those made by DFAs. This often results in better descriptions of properties by hybrid functionals compared to standard DFAs. However, in the case of the VDE of water cluster anions, the popular global hybrid B3LYP gives no improvement. In

fact, it can be seen from Table 2 that the MAE with B3LYP (238 meV) is comparable to that with PBE, which sits two rungs lower on Jacob's ladder. The performance of SCAN is substantially better than B3LYP. This may be due to the correlation component of the SCAN functional being self-interaction free. It is consistent with recent reports ${ }^{45546}$ that show SCAN provides a much improved description of liquid water and neutral water clusters than other DFAs, and with the fact that SCAN gives the correct ordering of water cluster anion isomers ordering as discussed above.

The relatively poor performance of B3LYP despite having an admixture of HF exchange 
is perplexing. The over-binding tendency of B3LYP, or, in other words, the effect of mixing HF with DFAs on the electronic properties, was investigated by Yagi and coworkers ${ }^{41}$. These authors studied water cluster anions using the long-range corrected DFAs (LC-DFAs). In this approximation, the electron repulsion operator is divided into short-range and long range parts with the short range part being described by the DFA and the long-range orbitalorbital exchange interaction described using HF exchange. They used the BLYP $\frac{78179}{17}$ and $\mathrm{BOP}^{78800}$ functionals for the short-range. Their results showed that both the LC-BLYP and LC-BOP functionals provided significant improvement in predicting VDEs over the global hybrid B3LYP. They attributed the excellent performance of the LC-BOP functional (MAE $35 \mathrm{meV}$ ) over the LC-BLYP functional (MAE $152 \mathrm{meV}$ ) to the satisfaction of fundamental conditions by the BOP functional and violation of them by BLYP. Their results show that good estimates of VDE comparable to those of MP2 can be obtained using long range corrected functionals.

It is interesting to compare the performance of these range-corrected functionals with the present approach where the self-interaction is explicitly removed on an orbital by orbital basis using the Perdew-Zunger method. We find that the removal of self-interaction error significantly improves the VDE obtained from the total energy differences of anion and neutral clusters. The VDEs obtained from the total energy differences for the SIC-LSDA, SIC-PBE, and SIC-SCAN functionals are summarized in Table2. The MAE in VDEs for the three SIC-DFAs are $180 \mathrm{meV}$ for SIC-LSDA, 60 for SIC-PBE, and $57 \mathrm{meV}$ for SIC-SCAN. These errors can be compared to those for the uncorrected functionals - $339 \mathrm{meV}$ for LSDA, $247 \mathrm{meV}$ for PBE, and 127 for the SCAN. This indicates that the SIE is a large contributor to the over-binding tendency of these approximations.

To better understand the nature of these errors, we used the self-interaction corrected densities obtained in the SIC-DFA calculations to evaluate the uncorrected DFAs. We then used these to recompute the VDEs. Following the notation adopted in the literature, this approach is called DFA@SIC-DFA ${ }^{81}$. Using this scheme, (Cf. Table 3) we find that the MAE 
in LSDA@SIC-LSDA, PBE@SIC-PBE, and SCAN@SIC-SCAN to be 303, 217, and $110 \mathrm{meV}$, respectively. This is only a slight $(<15 \%)$ improvement over the self-consistent DFA values. The primary source of the errors in VDEs therefore is the approximate functional and not simply the density. Burke and coworkers ${ }^{[22}$ categorized DFT calculations as either normal or abnormal depending on whether errors stem from the approximate functional (normal) or from the approximate density (abnormal). The comparison of VDES from the DFAs, and DFAs@SIC-DFAs show that the VDE calculations are apparently normal.

One drawback of the pure DFAs (LSDA, PBE, and SCAN) discussed above is that although the total energy of the water cluster is correctly lowered with the addition of an extra electron, the eigenvalue of the extra electron is positive within these approximations. The over-binding tendency of the DFAs discussed earlier is for VDE estimates made from the total energy difference between corresponding anion and neutral clusters. In exact DFT, the highest occupied eigenvalue equals the negative of the ionization potential ${ }^{71 / 74}$. This relationship does not strictly hold for approximate density functionals and in most DFAs, the absolute value of the HOMO eigenvalue substantially underestimates the first ionization potential due to self-interaction error. The positive eigenvalue of the extra electron in the water anions in DFT indicates that the extra electron is not actually bound in the complete basis set limit. The positive eigenvalue is a result of self-interaction error which makes the asymptotic potential seen by the electron positive. Removing self-interaction improves the asymptotic description of the potential and results in negative (bound) eigenvalues for the extra electron in all three SIC-DFAs used in this work. The improved description of the binding of the extra electron due to self-interaction correction can be seen from Table 2 which presents predictions of the VDE from the eigenvalues of the extra electron in the SIC-DFA calculations $\left(\mathrm{VDE}=-\epsilon_{\text {НОмо }}\right)$. The eigenvalues are excellent approximations to the VDEs, especially for SIC-PBE, for which the MAE with respect to $\operatorname{CCSD}(\mathrm{T})$ estimates is only 17 meV. The MAE for SIC-SCAN and SIC-LSDA eigenvalues are 44 and $117 \mathrm{meV}$, respectively. It is not obvious why the SIC-PBE eigenvalues approximate CCSD(T) VDE better than 
SIC-SCAN or SIC-LSDA. The SIC-SCAN HOMO eigenvalues agree better with the available experimental VDE values, with a MUE of $29 \mathrm{meV}$ compared to $35 \mathrm{meV}$ for SIC-PBE. The SIC-LDA eigenvalues have a MUE of $70 \mathrm{meV}$ when compared with the experimental values. For all three functionals, the SIC-DFA HOMO eigenvalues are better approximations of the VDEs than the total energy differences. The shift of the anion eigenvalues to positions close to the removal energies underscores that the self-interaction correction is needed for a more physically correct description of water cluster anions with DFAs.

It should be noted that the positive HOMO eigenvalues in the uncorrected DFA calculations imply that the over-binding of the VDE by DFA total energy differences is actually worse than seen in Table 2. The energy of the cluster anion would be lowered by removing a fraction of the extra electron to a large distance from the cluster. The minimum energy state corresponds to removing sufficient charge to make the HOMO eigenvalue zero. Lower anion energies would give still large VDEs than those in Table 2.

\section{Conclusion}

We have used the recently developed Fermi-Löwdin orbital self-interaction correction scheme with the LSDA, PBE, and SCAN meta-GGA functionals to study small water cluster anions. Our results show that the SCAN functional provides a very good description of isomer ordering, as well as the relative energies of isomers, when compared to $\operatorname{CCSD}(\mathrm{T})$ results. The application of FLOSIC significantly improves the agreement for SIC-LSDA and SIC-PBE relative to $\operatorname{CCSD}(\mathrm{T})$, however, the SIC-SCAN results deviate somewhat more from the reference values than SCAN. The excellent performance of SCAN for binding energies does not carry over to the description of the binding of the extra electron. The SCAN MAE (127 meV) for electron detachment energy, although smallest among the LSDA (339 meV), PBE GGA $(247 \mathrm{meV})$ and also earlier reported B3LYP $(238 \mathrm{meV})$ results, is still substantially larger than the MP2 MAE (44 meV). Removing self-interaction results in significantly improved 
Table 2: Vertical detachment energies and HOMO eigenvalues in meV of water cluster anions. The mean absolute deviation (MAD) is respect to the CCSD(T) values. The uncertainties of the experimental values are about $\pm 30 \mathrm{meV}$.

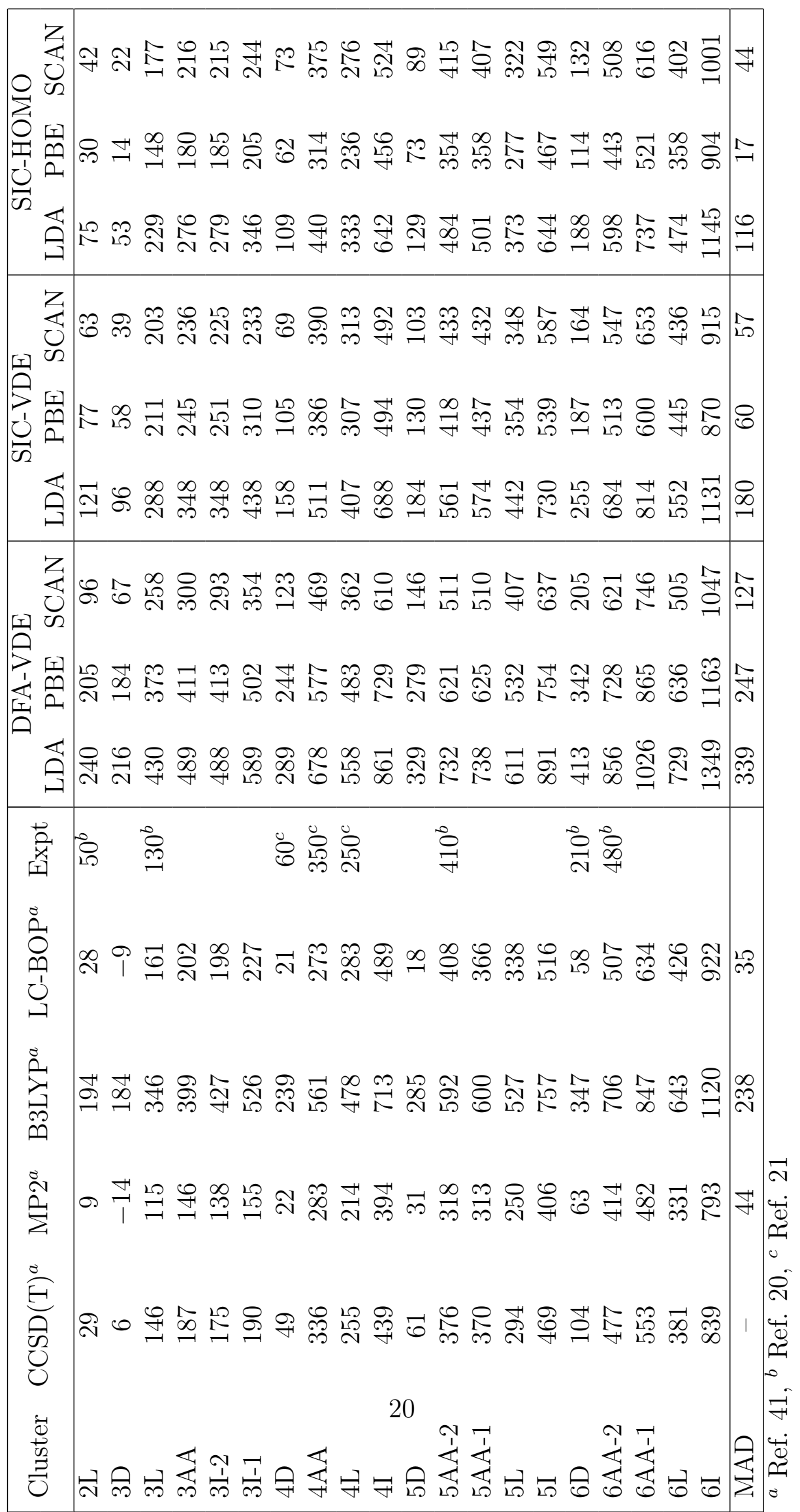


Table 3: VDEs (in meV) obtained using DFA total energies computed with selfconsistent FLOSIC-DFA densities (DFA@FLOSIC) compared to the reported VDEs obtained with CCSD(T). The mean absolute deviation (MAD) is respect to the $\operatorname{CCSD}(\mathrm{T})$ values.

\begin{tabular}{lcccc}
\hline \hline Cluster & LDA & PBE & SCAN & ${\text { CCSD }(T)^{a}}^{a}$ \\
\hline 2L & 217 & 184 & 82 & 29 \\
3D & 191 & 165 & 58 & 6 \\
3L & 403 & 303 & 237 & 146 \\
3AA & 456 & 383 & 280 & 187 \\
3I-2 & 389 & 389 & 275 & 175 \\
3I-1 & 467 & 468 & 327 & 190 \\
4D & 264 & 223 & 111 & 49 \\
4AA & 651 & 545 & 446 & 336 \\
4L & 532 & 453 & 340 & 255 \\
4I & 844 & 704 & 584 & 439 \\
5D & 299 & 248 & 114 & 61 \\
5AA-2 & 712 & 586 & 547 & 376 \\
5AA-1 & 699 & 593 & 493 & 370 \\
5L & 581 & 496 & 386 & 294 \\
5I & 873 & 725 & 627 & 469 \\
6D & 380 & 311 & 190 & 104 \\
6AA-2 & 832 & 697 & 601 & 477 \\
6AA-1 & 983 & 808 & 720 & 553 \\
6L & 695 & 601 & 484 & 381 \\
6I & 1331 & 1142 & 1024 & 839 \\
\hline MAD & 303 & 217 & 110 & - \\
\hline \hline
\end{tabular}

${ }^{a}$ Ref. 41 
VDEs for all functionals, with about $60 \mathrm{meV}$ errors for the SIC-SCAN and SIC-PBE. Similarly, removing self-interaction is essential for obtaining orbital energies that are consistent with electron binding. For SIC-PBE, the HOMO eigenvalues give remarkably good predictions of VDEs, with a MAE with respect to $\mathrm{CCSD}(\mathrm{T})$ of only $17 \mathrm{meV}$. An interesting feature of the FLOSIC calculations is the chemical insight that can be gained from the Fermi-orbital descriptor (FOD) positions. The FOD associated with the extra electron indicates where the the excess charge is accommodated in the clusters. In several cases, the FOD position is relatively far away from the cluster center, indicating a more delocalized density for the extra electon.

\section{Acknowledgement}

The authors acknowledge useful discussions with Dr. Luis Basurto. This work was supported by the US Department of Energy, Office of Science, Office of Basic Energy Sciences, as part of the Computational Chemical Sciences Program under Award No. de-sc0018331. The work of R.R.Z. was supported in part by the US Department of Energy, Office of Science, Office

of Basic Energy Sciences, under Award No. de-sc0006818. Support for computational time at the Texas Advanced Computing Center through NSF Grant No. TG-DMR090071, and at NERSC is gratefully acknowledged.

\section{References}

(1) Hart, E. J. In Survey of Progress in Chemistry; Scott, A. F., Ed.; Survey of Progress in Chemistry; Elsevier, 1969; Vol. 5; pp 129-184.

(2) Garrett, B. C.; Dixon, D. A.; Camaioni, D. M.; Chipman, D. M.; Johnson, M. A.; Jonah, C. D.; Kimmel, G. A.; Miller, J. H.; Rescigno, T. N.; Rossky, P. J. et al. Role 
of Water in Electron-Initiated Processes and Radical Chemistry: Issues and Scientific Advances. Chemical Reviews 2005, 105, 355-390.

(3) Kulkarni, S. A.; Bartolotti, L. J.; Pathak, R. K. Ab initio studies of anionic clusters of water pentamer. The Journal of Chemical Physics 2000, 113, 2697-2700.

(4) Rai, D.; Kulkarni, A. D.; Gejji, S. P.; Pathak, R. K. Water Clusters (H2O)n, n=6-8, in External Electric Fields. The Journal of Chemical Physics 2008, 128, 034310.

(5) Herbert, J. M. The quantum chemistry of loosely bound electrons. Reviews in Computational Chemistry 2015, 28, 391-517.

(6) Herbert, J. M.; Coons, M. P. The hydrated electron. Annual review of physical chemistry $\mathbf{2 0 1 7}, 68,447-472$.

(7) Grooß, J.-U.; Müller, R. Do cosmic-ray-driven electron-induced reactions impact stratospheric ozone depletion and global climate change? Atmospheric Environment 2011, 45, 3508-3514.

(8) Alizadeh, E.; Orlando, T. M.; Sanche, L. Biomolecular Damage Induced by Ionizing Radiation: The Direct and Indirect Effects of Low-Energy Electrons on DNA. Annual Review of Physical Chemistry 2015, 66, 379-398.

(9) Armbruster, M.; Haberland, H.; Schindler, H.-G. Negatively Charged Water Clusters, or the First Observation of Free Hydrated Electrons. Phys. Rev. Lett. 1981, 47, 323326.

(10) Hammer, N. I.; Shin, J.-W.; Headrick, J. M.; Diken, E. G.; Roscioli, J. R.; Weddle, G. H.; Johnson, M. A. How Do Small Water Clusters Bind an Excess Electron? Science 2004, 306, 675-679.

(11) Turi, L.; Sheu, W.-S.; Rossky, P. J. Characterization of Excess Electrons in WaterCluster Anions by Quantum Simulations. Science 2005, 309, 914-917. 
(12) Jacobson, L. D.; Herbert, J. M. Theoretical Characterization of Four Distinct Isomer Types in Hydrated-Electron Clusters, and Proposed Assignments for Photoelectron Spectra of Water Cluster Anions. Journal of the American Chemical Society 2011, 133, 19889-19899.

(13) Rossky, P. J.; Schnitker, J. The hydrated electron: quantum simulation of structure, spectroscopy, and dynamics. The Journal of Physical Chemistry 1988, 92, 4277-4285.

(14) Turi, L.; Rossky, P. J. Theoretical Studies of Spectroscopy and Dynamics of Hydrated Electrons. Chemical Reviews 2012, 112, 5641-5674.

(15) Wilhelm, J.; VandeVondele, J.; Rybkin, V. V. Dynamics of the Bulk Hydrated Electron from Many-Body Wave-Function Theory. Angewandte Chemie International Edition 2019, 58, 3890-3893.

(16) Feng, D.-F.; Kevan, L. Theoretical models for solvated electrons. Chemical Reviews 1980, 80, 1-20.

(17) Kevan, L. Solvated electron structure in glassy matrixes. Accounts of Chemical Research $1981,14,138-145$.

(18) Coe, J. V.; Lee, G. H.; Eaton, J. G.; Arnold, S. T.; Sarkas, H. W.; Bowen, K. H.; Ludewigt, C.; Haberland, H.; Worsnop, D. R. Photoelectron spectroscopy of hydrated electron cluster anions, $(\mathrm{H} 2 \mathrm{O}) \mathrm{n}-\mathrm{n}=2-69$. The Journal of Chemical Physics 1990, 92, 3980-3982.

(19) Kim, K. S.; Park, I.; Lee, S.; Cho, K.; Lee, J. Y.; Kim, J.; Joannopoulos, J. D. The Nature of a Wet Electron. Phys. Rev. Lett. 1996, 76, 956-959.

(20) Kim, J.; Becker, I.; Cheshnovsky, O.; Johnson, M. A. Photoelectron spectroscopy of the 'missing' hydrated electron clusters $(\mathrm{H} 2 \mathrm{O})-\mathrm{n}, \mathrm{n}=3,5,8$ and 9: Isomers and continuity 
with the dominant clusters $\mathrm{n}=6,7$ and $>$ 11. Chemical Physics Letters 1998, 297, 90 $-96$.

(21) Shin, J.-W.; Hammer, N. I.; Headrick, J. M.; Johnson, M. A. Preparation and photoelectron spectrum of the 'missing' (H2O)4- cluster. Chemical Physics Letters 2004, 399, $349-353$.

(22) Verlet, J. R. R.; Bragg, A. E.; Kammrath, A.; Cheshnovsky, O.; Neumark, D. M. Observation of Large Water-Cluster Anions with Surface-Bound Excess Electrons. Science 2005, 307, 93-96.

(23) Coe, J. V.; Williams, S. M.; Bowen, K. H. Photoelectron spectra of hydrated electron clusters vs. cluster size: connecting to bulk. International Reviews in Physical Chemistry 2008, 27, 27-51.

(24) Ma, L.; Majer, K.; Chirot, F.; von Issendorff, B. Low temperature photoelectron spectra of water cluster anions. The Journal of Chemical Physics 2009, 131, 144303.

(25) Bailey, C. G.; Kim, J.; Johnson, M. A. Infrared Spectroscopy of the Hydrated Electron Clusters (H2O)n-, $\mathrm{n}=6$, 7: Evidence for Hydrogen Bonding to the Excess Electron. The Journal of Physical Chemistry 1996, 100, 16782-16785.

(26) Ayotte, P.; Weddle, G. H.; Bailey, C. G.; Johnson, M. A.; Vila, F.; Jordan, K. D. Infrared spectroscopy of negatively charged water clusters: Evidence for a linear network. The Journal of Chemical Physics 1999, 110, 6268-6277.

(27) Diken, E. G.; Robertson, W. H.; Johnson, M. A. The Vibrational Spectrum of the Neutral (H2O)6 Precursor to the "Magic" (H2O)6- Cluster Anion by Argon-Mediated, Population-Modulated Electron Attachment Spectroscopy. The Journal of Physical Chemistry A 2004, 108, 64-68. 
(28) Asmis, K. R.; Santambrogio, G.; Zhou, J.; Garand, E.; Headrick, J.; Goebbert, D.; Johnson, M. A.; Neumark, D. M. Vibrational spectroscopy of hydrated electron clusters $(\mathrm{H} 2 \mathrm{O})_{15-50}^{-}$via infrared multiple photon dissociation. The Journal of Chemical Physics 2007, 126, 191105.

(29) Guasco, T. L.; Elliott, B. M.; Johnson, M. A.; Ding, J.; Jordan, K. D. Isolating the Spectral Signatures of Individual Sites in Water Networks Using Vibrational DoubleResonance Spectroscopy of Cluster Isotopomers. The Journal of Physical Chemistry Letters 2010, 1, 2396-2401.

(30) Hammer, N. I.; Roscioli, J. R.; Johnson, M. A.; Myshakin, E. M.; Jordan, K. D. Infrared Spectrum and Structural Assignment of the Water Trimer Anion. The Journal of Physical Chemistry A 2005, 109, 11526-11530.

(31) Hammer, N. I.; Roscioli, J. R.; Johnson, M. A. Identification of Two Distinct Electron Binding Motifs in the Anionic Water Clusters: A Vibrational Spectroscopic Study of the (H2O)6- Isomers. The Journal of Physical Chemistry A 2005, 109, 7896-7901.

(32) Choi, T. H.; Jordan, K. D. Potential energy landscape of the (H2O)6- cluster. Chemical Physics Letters 2009, 475, 293 - 297.

(33) Lee, S.; Lee, S. J.; Lee, J. Y.; Kim, J.; Kim, K. S.; Park, I.; Cho, K.; Joannopoulos, J. Ab initio study of water hexamer anions. Chemical Physics Letters 1996, 254, 128 134 .

(34) Tsurusawa, T.; Iwata, S. Theoretical studies of the water-cluster anions containing the OHeHO structure: energies and harmonic frequencies. Chemical Physics Letters 1999, $315,433-440$.

(35) Kim, J.; Suh, S. B.; Kim, K. S. Water dimer to pentamer with an excess electron: Ab initio study. The Journal of Chemical Physics 1999, 111, 10077-10087. 
(36) Weigend, F.; Ahlrichs, R. Abinitio treatment of (H2O)2- and (H2O)6-. Phys. Chem. Chem. Phys. 1999, 1, 4537-4540.

(37) Lee, H. M.; Lee, S.; Kim, K. S. Structures, energetics, and spectra of electron-water clusters, e-(H2O)2-6 and e-HOD(D2O)1-5. The Journal of Chemical Physics 2003, 119, 187-194.

(38) Lee, H. M.; Suh, S. B.; Tarakeshwar, P.; Kim, K. S. Origin of the magic numbers of water clusters with an excess electron. The Journal of Chemical Physics 2005, 122, 044309.

(39) Herbert, J. M.; Head-Gordon, M. Calculation of Electron Detachment Energies for Water Cluster Anions: An Appraisal of Electronic Structure Methods, with Application to (H2O)20- and (H2O)24-. The Journal of Physical Chemistry A 2005, 109, 5217-5229.

(40) Herbert, J. M.; Head-Gordon, M. Accuracy and limitations of second-order many-body perturbation theory for predicting vertical detachment energies of solvated-electron clusters. Phys. Chem. Chem. Phys. 2006, 8, 68-78.

(41) Yagi, K.; Okano, Y.; Sato, T.; Kawashima, Y.; Tsuneda, T.; Hirao, K. Water Cluster Anions Studied by the Long-Range Corrected Density Functional Theory. The Journal of Physical Chemistry A 2008, 112, 9845-9853.

(42) Ünal, A.; Bozkaya, U. Anionic water pentamer and hexamer clusters: An extensive study of structures and energetics. The Journal of Chemical Physics 2018, 148, 124307.

(43) Zho, C.-C.; Vlček, V.; Neuhauser, D.; Schwartz, B. J. Thermal Equilibration Controls H-Bonding and the Vertical Detachment Energy of Water Cluster Anions. The Journal of Physical Chemistry Letters 2018, 9, 5173-5178.

(44) Becke, A. D. A new mixing of Hartree-Fock and local density functional theories. The Journal of Chemical Physics 1993, 98, 1372-1377. 
(45) Sun, J.; Ruzsinszky, A.; Perdew, J. P. Strongly Constrained and Appropriately Normed Semilocal Density Functional. Phys. Rev. Lett. 2015, 115, 036402.

(46) Chen, M.; Ko, H.-Y.; Remsing, R. C.; Andrade, M. F. C.; Santra, B.; Sun, Z.; Selloni, A.; Car, R.; Klein, M. L.; Perdew, J. P. et al. Ab initio theory and modeling of water. Proceedings of the National Academy of Sciences 2017, 114, 10846-10851.

(47) Perdew, J. P.; Burke, K.; Ernzerhof, M. Generalized Gradient Approximation Made Simple. Phys. Rev. Lett. 1996, 77, 3865-3868.

(48) Perdew, J. P.; Zunger, A. Self-interaction correction to density-functional approximations for many-electron systems. Phys. Rev. B 1981, 23, 5048-5079.

(49) Pederson, M. R.; Heaton, R. A.; Lin, C. C. Local-density Hartree-Fock theory of electronic states of molecules with self-interaction correction. The Journal of Chemical Physics 1984, 80, 1972-1975.

(50) Pederson, M. R.; Heaton, R. A.; Lin, C. C. Density functional theory with selfinteraction correction: Application to the lithium molecules). The Journal of Chemical Physics 1985, 82, 2688-2699.

(51) Pederson, M. R.; Ruzsinszky, A.; Perdew, J. P. Communication: Self-interaction correction with unitary invariance in density functional theory. The Journal of Chemical Physics 2014, 140, 121103.

(52) Löwdin, P. On the Non-Orthogonality Problem Connected with the Use of Atomic Wave Functions in the Theory of Molecules and Crystals. The Journal of Chemical Physics 1950, 18, 365-375.

(53) Pederson, M. R. Fermi orbital derivatives in self-interaction corrected density functional theory: Applications to closed shell atoms. The Journal of Chemical Physics 2015, 142, 064112. 
(54) Pederson, M. R.; Baruah, T. In Chapter Eight-Self-Interaction Corrections Within the Fermi-Orbital-Based Formalism; Arimondo, E., Lin, C. C., Yelin, S. F., Eds.; Advances In Atomic, Molecular, and Optical Physics; Academic Press, 2015; Vol. 64; pp 153 180.

(55) Liu, D. C.; Nocedal, J. On the limited memory BFGS method for large scale optimization. Mathematical programming 1989, 45, 503-528.

(56) Yamamoto, Y.; Diaz, C. M.; Basurto, L.; Jackson, K. A.; Baruah, T.; Zope, R. R. Fermi-Löwdin orbital self-interaction correction using the strongly constrained and appropriately normed meta-GGA functional. The Journal of Chemical Physics 2019, 151, 154105.

(57) Baruah, T.; Yamamoto, Y.; Basurto, L.; Diaz, C. M.; Zope, R. R. Self-interaction correction to density functional approximations using Fermi-Löwdin orbitals: Methodology and Parallelization. (unpublished).

(58) Jackson, K.; Pederson, M. R. Accurate forces in a local-orbital approach to the localdensity approximation. Phys. Rev. B 1990, 42, 3276-3281.

(59) Pederson, M.; Porezag, D.; Kortus, J.; Patton, D. Strategies for Massively Parallel Local-Orbital-Based Electronic Structure Methods. physica status solidi (b) 2000, 217, $197-218$.

(60) Porezag, D.; Pederson, M. R. Optimization of Gaussian basis sets for density-functional calculations. Phys. Rev. A 1999, 60, 2840-2847.

(61) Pederson, M. R.; Jackson, K. A. Variational mesh for quantum-mechanical simulations. Phys. Rev. B 1990, 41, 7453-7461.

(62) Kao, D.-y.; Withanage, K.; Hahn, T.; Batool, J.; Kortus, J.; Jackson, K. Self-consistent self-interaction corrected density functional theory calculations for atoms using Fermi- 
Löwdin orbitals: Optimized Fermi-orbital descriptors for Li-Kr. The Journal of Chemical Physics 2017, 147, 164107.

(63) Withanage, K. P.; Trepte, K.; Peralta, J. E.; Baruah, T.; Zope, R.; Jackson, K. A. On the Question of the Total Energy in the Fermi-Löwdin Orbital Self-Interaction Correction Method. Journal of chemical theory and computation 2018, 14, 4122-4128.

(64) Pederson, M. R.; Baruah, T.; Kao, D.-y.; Basurto, L. Self-interaction corrections applied to Mg-porphyrin, $\mathrm{C}_{60}$, and pentacene molecules. The Journal of Chemical Physics 2016, $144,164117$.

(65) Sharkas, K.; Li, L.; Trepte, K.; Withanage, K. P.; Joshi, R. P.; Zope, R. R.; Baruah, T.; Johnson, J. K.; Jackson, K. A.; Peralta, J. E. Shrinking Self-Interaction Errors with the Fermi-Löwdin Orbital Self Interaction Corrected Density Functional Approximation. The Journal of Physical Chemistry A 2018, 122, 9307-9315.

(66) Shahi, C.; Bhattarai, P.; Wagle, K.; Santra, B.; Schwalbe, S.; Hahn, T.; Kortus, J.; Jackson, K. A.; Peralta, J. E.; Trepte, K. et al. Stretched or noded orbital densities and self-interaction correction in density functional theory. The Journal of Chemical Physics 2019, 150, 174102.

(67) Kao, D.-y.; Pederson, M.; Hahn, T.; Baruah, T.; Liebing, S.; Kortus, J. The Role of Self-Interaction Corrections, Vibrations, and Spin-Orbit in Determining the Ground Spin State in a Simple Heme. Magnetochemistry 2017, 3, 31.

(68) Joshi, R. P.; Trepte, K.; Withanage, K. P.; Sharkas, K.; Yamamoto, Y.; Basurto, L.; Zope, R. R.; Baruah, T.; Jackson, K. A.; Peralta, J. E. Fermi-Löwdin orbital selfinteraction correction to magnetic exchange couplings. The Journal of Chemical Physics 2018, 149, 164101.

(69) Johnson, A. I.; Withanage, K. P.; Sharkas, K.; Yamamoto, Y.; Baruah, T.; Zope, R. R.; Peralta, J. E.; Jackson, K. A. The effect of self-interaction error on electrostatic dipoles 
calculated using density functional theory. The Journal of Chemical Physics 2019, 151, 174106.

(70) Withanage, K. P.; Akter, S.; Shahi, C.; Joshi, R. P.; Diaz, C.; Yamamoto, Y.; Zope, R.; Baruah, T.; Perdew, J. P.; Peralta, J. E. et al. Self-interaction-free electric dipole polarizabilities for atoms and their ions using the Fermi-Löwdin self-interaction correction. Physical Review A 2019, 100, 012505.

(71) Perdew, J. P.; Parr, R. G.; Levy, M.; Balduz Jr, J. L. Density-functional theory for fractional particle number: derivative discontinuities of the energy. Physical Review Letters 1982, 49, 1691.

(72) Levy, M.; Perdew, J. P.; Sahni, V. Exact differential equation for the density and ionization energy of a many-particle system. Physical Review A 1984, 30, 2745.

(73) Perdew, J. P.; Levy, M. Comment on "Significance of the highest occupied Kohn-Sham eigenvalue". Physical Review B 1997, 56, 16021.

(74) Harbola, M. K. Relationship between the highest occupied Kohn-Sham orbital eigenvalue and ionization energy. Phys. Rev. B 1999, 60, 4545-4550.

(75) Odutola, J. A.; Dyke, T. R. Partially deuterated water dimers: Microwave spectra and structure. The Journal of Chemical Physics 1980, 72, 5062-5070.

(76) Sharkas, K.; Wagle, K.; Santra, B.; Akter, S.; Zope, R. R.; Baruah, T.; Jackson, K.; Perdew, J. P.; Peralta, J. preprint.

(77) Perdew, J. P.; Schmidt, K. Jacob's ladder of density functional approximations for the exchange-correlation energy. AIP Conference Proceedings. 2001; pp 1-20.

(78) Becke, A. D. Density functional exchange energy approximation with correct asymptotic behavior. Phys. Rev. A 1988, 38, 3098-3100. 
(79) Lee, C.; Yang, W.; Parr, R. G. Development of the Colle-Salvetti correlation-energy formula into a functional of the electron density. Phys. Rev. B 1988, 37, 785-789.

(80) Tsuneda, T.; Suzumura, T.; Hirao, K. A reexamination of exchange energy functionals. The Journal of Chemical Physics 1999, 111, 5656-5667.

(81) Verma, P.; Perera, A.; Bartlett, R. J. Increasing the applicability of DFT I: Nonvariational correlation corrections from Hartree-Fock DFT for predicting transition states. Chemical Physics Letters 2012, 524, 10-15.

(82) Kim, M.-C.; Sim, E.; Burke, K. Understanding and reducing errors in density functional calculations. Phys. Rev. Lett. 2013, 111, 073003. 